\title{
Brieftauben und ein verschollenes Teestrauchgewächs - wie der Botanische Garten Frankfurt am Main zu einer Pflanzenrarität kam
}

\author{
Manfred Wessel
}

\begin{abstract}
Franklinia alatamaha is an autumnal attraction in the Botanical Garden Frankfurt am Main. The history of this plant, ecology and botany will be illustrated and the interesting episodes of the life of Prof. Dr. KLaus Schmidt-Koenig in Frankfurt am Main will be introduced.
\end{abstract}

\section{Zusammenfassung}

Franklinia alatamaha ist eine herbstliche Attraktion im Botanischen Garten Frankfurt am Main. Die Geschichte dieser Pflanze, ihre Ökologie und Botanik werden erläutert und die interessanten Episoden der Frankfurter Zeit von Prof. Dr. KLAus Schmidt-Koenig werden vorgestellt.

\section{Einleitung}

Profession und Leidenschaft sind zum Bedauern vieler werktätiger Menschen nicht immer deckungsgleich, aber auch nicht unbedingt wesensfremd zueinander. Diesem Umstand ist es zu verdanken, dass sich die Besucherinnen und Besucher des Botanischen Gartens Frankfurt am Main an einem Gehölz erfreuen können, welches in der Natur am heimatlichen Standort als verschollen, wenn nicht sogar ausgestorben gilt. Das Gehölz, von dem hier die Rede sein soll, heißt Franklinie oder auch FrankuinBaum. Diese schöne und attraktive Pflanze verdankt der Botanische Garten einem zu seinen

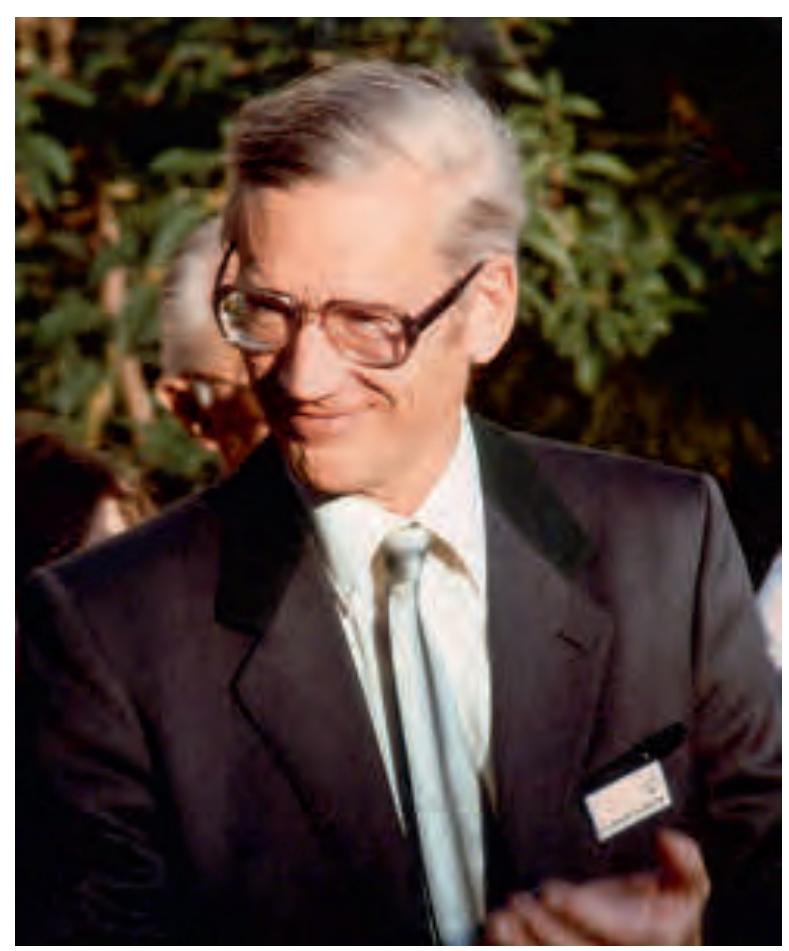

Lebzeiten sehr aktiven Mitglied der Deutschen Dendrologischen Gesellschaft (seine Leidenschaft), der im Brotberuf (seine Profession) ein Wissenschaftler gewesen ist, der seine bedeutendsten Forschungsergebnisse auf dem Gebiet der Verhaltensforschung bei Brieftauben erreichte: Klaus Schmidt-Koenig. Wie dies alles nun zusammen ein stimmiges Bild ergibt, wird nachfolgend berichtet. Auch Interessantes zur Geschichte und Botanik der sagenhaften Pflanzenart wird dargestellt.

\section{Klaus Schmidt-Koenig: Zoologe, Ornithologe, Verhaltensphysiologe und Dendrologe}

Schmidt-Koenig erblickte das Licht der Welt am 21.01.1930 in Heidelberg. Bereits als Schüler interessierte ihn die Ornithologie sehr. Nach dem Abitur in Heidelberg und einem begonnenen Chemie-Studium in München wechselte er das Studienfach und studierte Biologie in Heidelberg, wo er 1958 mit „summa cum laude“ promoviert wurde. Die anschließende Tätigkeit an der Duke University in Durham, North Carolina (USA), führte ihn bereits in die Nähe des ehemals heimatlichen Standorts unserer Franklinie, der im südlich an North Carolina angrenzenden Bundesstaat Georgia lag. Eine kurze Unterbrechung seiner wissenschaftlichen

Abb. 1: Klaus Schmidt-Koenig, Aufnahme aus dem Zeitraum I996-I998. 
Tätigkeit in Durham führte ihn ins niedersächsische Wattenmeer auf die Nordsee-Insel Wangerooge, wo er als Vogelwart arbeitete, bevor er in die USA zurückging. Heimgekehrt nach Deutschland, lehrte und forschte SchмidTKoenig von 1963 bis 1975 an der Universität Göttingen, bevor er 1975 bis zu seiner Emeritierung 1996 an die Universität Tübingen wechselte. In Tübingen hatte er den Lehrstuhl für Verhaltensphysiologie inne und war seit 1971 außerdem Professor of Zoology an der Duke University in Durham, wo seine wissenschaftliche Karriere begonnen hatte. Klaus SchmidtKoenig verstarb in seinem Haus in Oberkirch (Baden-Württemberg) im Kreise seiner Familie im Alter von 79 Jahren am 18.02.2009.

Schmidt-Koenig hatte in seiner Dissertation bei Gustav Kramer die Grundlagen für den Nachweis der Verwendung des Sonnenkompasses durch Tauben erarbeitet. Um den Sinn dieser Forschungsarbeit zu verstehen, ist ein Blick in die Geschichte der Brieftauben von Nutzen. Brieftauben besitzen eine lange Tradition in der Nachrichtenübermittlung, denn sie benötigen dafür keine Straßen, Bahngleise oder Telefonleitungen. Als der Mensch erstmals realisierte, dass Tauben - in beliebiger Entfernung zu ihrem Heimatschlag ausgesetzt - scheinbar wie durch magnetische Kräfte angezogen diesen wieder ansteuern und damit zurückkehren, war die Basis für eine Erfolgsgeschichte gelegt. Diese führte im zivilen Bereich zu sportlichen Wettkämpfen und der Gründung zahlreicher Brieftaubenzuchtvereine. Zentrum in Deutschland war hier seit den Anfängen in den 1860er Jahren das Ruhrgebiet. In Essen residiert folgerichtig der Verband Deutscher Brieftaubenzüchter e. V., und hier entstand auch die Bezeichnung der Brieftaube als „Rennpferd des kleinen (Berg-)Mannes“. Aber auch sehr viel traurigere Einsatzfelder wurden durch die Brieftaube abgedeckt. Schon Pharao Ramses III. nutzte sie als zuverlässige Botenvögel und

Abb. 2: Junge Frau in orientalischer Kleidung mit Brieftaube (Ölgemälde von einem anonymen Meister). bis zu den Schlachtfeldern des Zweiten Weltkrieges waren sie im Einsatz. Die Nachricht der Niederlage Napoleons und seiner französischen Truppen in der Schlacht von Waterloo am 18. Juni 1815 wurde durch Brieftauben an die britische Regierung übermittelt. Im Grabenkrieg des Ersten Weltkriegs wurden Brieftauben auf deutscher und französischer Seite eingesetzt, wenn Telefon- und Telegrafenleitungen zerstört oder eine andersartige Übermittlung nicht möglich war. Doch wie schaffen Tauben eine Heimkehr aus über 1000 km Entfernung, freigelassen in ihnen fremdem Gelände und dabei Geschwindigkeiten von bis zu $100 \mathrm{~km}$ pro Stunde erreichend? Diese Frage beschäftigte viele Forscher, einer der erfolgreichsten und bedeutendsten von ihnen war KLAus Schmidt-Koenig. Er fand unter anderem heraus, dass Brieftauben wie auch Zugvögel den Stand der Sonne und der Sterne als Kompass verwenden können und dabei zusätzlich das Magnetfeld der Erde nutzen, also über zwei unabhängig voneinander arbeitende Systeme verfügen. Optische Anhaltspunkte zur Orientierung sind dabei unbedeutend, wie SснмidTKoenig nachwies, indem er seinen Tauben milchig-trübe Haftschalen, die das Blickfeld der Tiere entscheidend einschränkten, einsetzte

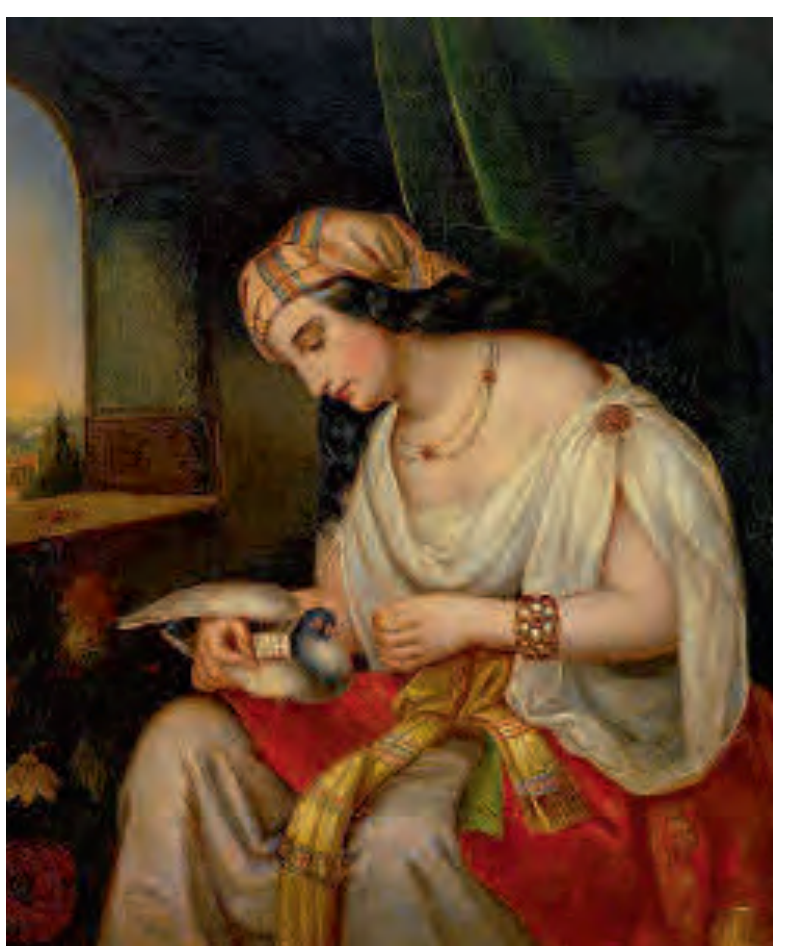




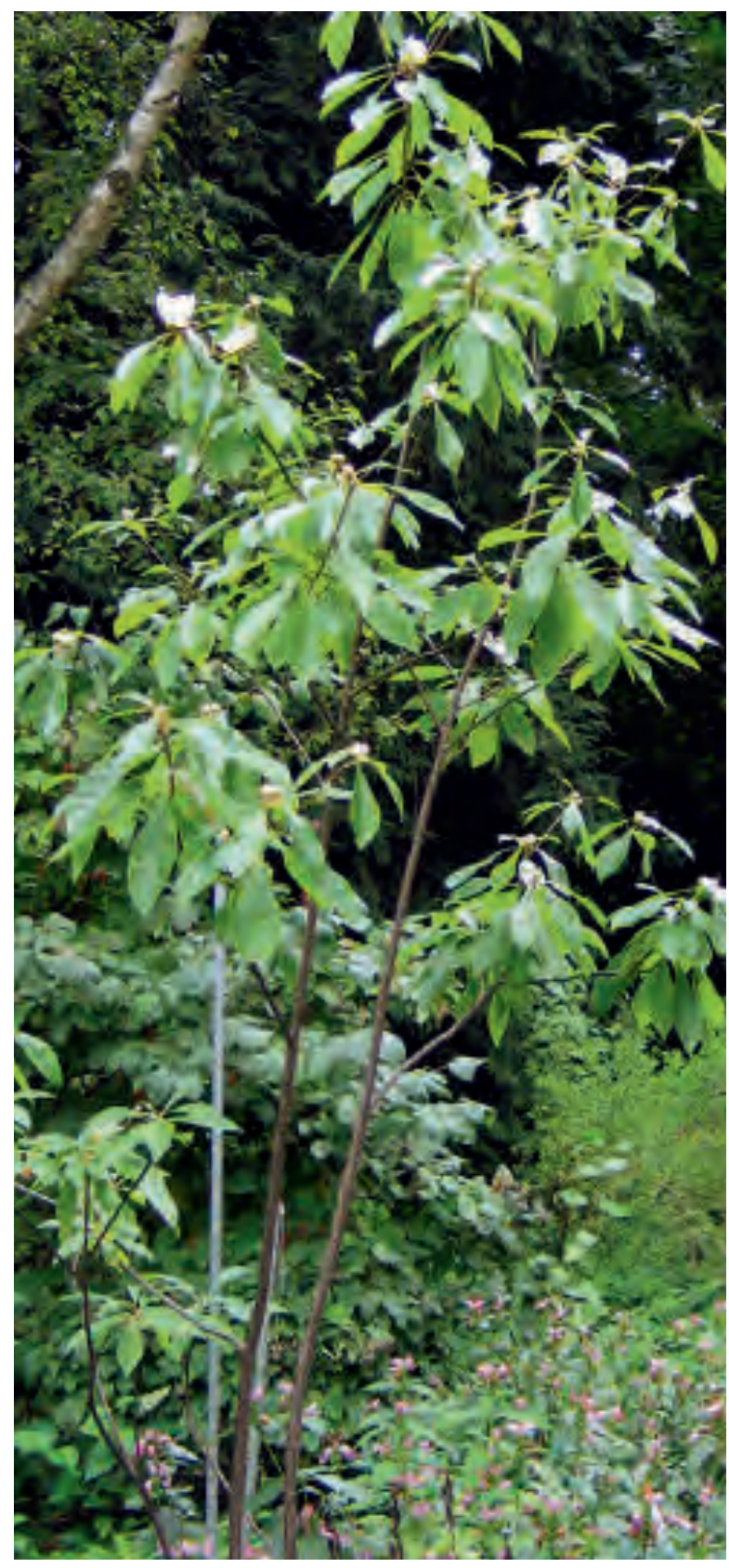

(Wiltschко \& Wiltschкo, mündl. Mitt.). Doch blieb er nicht bei den Tauben stehen, sondern dehnte sein Forscherinteresse auf z. B. den Monarchfalter aus, ein berühmter Wanderfalter, der bei seinen herbstlichen Wanderungen in Nordamerika bis zu 3500 Kilometer zurücklegen kann. Dieses Zugverhalten studierte Schmidt-Koenig über viele Jahre hinweg an der ostamerikanischen Küste und entwickelte dabei unter anderem den Einsatz belastbarer statistischer Verfahren in der Verhaltensforschung. Für seine Arbeiten zum Sonnenkompass erhielt Schmidt-Koenig 1995 den Orni-
thologen-Preis der Deutschen Ornithologischen Gesellschaft, deren Präsident er von 1986 bis 1991 war.

Neben der auch beruflich zu erklärenden Begeisterung für die Ornithologie war ScHMIDTKoenig ein ebenso begeisterter und sachkundiger Dendrologe und folglich Mitglied in der Deutschen Dendrologischen Gesellschaft. Von seinen Reisen nach Nord-Amerika brachte er immer wieder verschiedene Baum- und Straucharten mit nach Deutschland und pflanzte diese in seinem von ihm begründeten Arboretum im baden-württembergischen Oberkirch aus. Dieses auch von Fachleuten der Dendrologie gelobte und anerkannte Arboretum lag im Rheintal an einem westlich exponierten Weinbergshang in ca. $250 \mathrm{~m}$ Höhe in klimatisch begünstigter Lage. Mit einer Fläche von ca. 4 ha und über 1200 verschiedenen Akzessionen beherbergte es zahlreiche Raritäten der Gehölzflora und wurde oftmals Ziel von Exkursionen gehölzbegeisterter Menschen. Nach seinem Tod entwickelte sich allerdings die Zukunft des Arboretums bedauerlicherweise negativ, denn das gesamte Anwesen wurde familienseits verkauft und das Fortbestehen des „Arboretums Schmidt-Koenig" ist damit in Zukunft sehr in Frage gestellt (Junginger, mündl. Mitt.).

\section{Die Taubenschläge des Zoologischen Institutes der Goethe-Universität und die Geschichte von Franklinia alatamaha in Frankfurt am Main}

Klaus Schmidt-Koenig kam in den Jahren 1963/64 zusammen mit Mitarbeitern an Versuchstagen von Göttingen nach Frankfurt am Main, um hier einen Teil seiner Taubenversuche (die von ihm entwickelten KreuzschlagExperimente) durchzuführen. Dies hatte seinen einfachen Grund in der Nähe Göttingens, seines eigentlichen Standortes, zur damaligen Grenze der DDR, die mit nur zwischen 15 und

Abb. 3 (oben): Franklinia alatamaha, Habitus im Botanischen Garten (5.9.2008)

Abb. 4 (Seite 65): Rinde von Franklinia alatamaha. 
$20 \mathrm{~km}$ Entfernung seinen Versuchen eine unüberwindbare Barriere entgegenstellte, denn die Freisetzungsversuche wurden zur damaligen Zeit mit sehr viel weiteren Entfernungen konzipiert. Da passte es gut, dass ein ihm privat bekannter Taubenzüchter, der in der Verwaltung der Goethe-Universität arbeitete, die Verbindung zu den Zoologen in der Siesmayerstraße herstellte und Schmidt-Koenig dort auf offene Ohren stieß. Die Kontakte in Frankfurt entwickelten sich erfolgreich. Das Forscherehepaar Roswitha und Wolfgang Wiltschko gehörte zu seinem engen Freundes- und Kollegenkreis und ist heute ebenso bekannt aufgrund ihrer verwandten Forschungsarbeiten. Wolfgang Wiltschko gilt als einer der Pioniere auf dem Gebiet der Orientierung von Vögeln für seinen ersten Nachweis, dass sich Vögel zur Navigation auch am Magnetfeld der Erde orientieren. Das Ehepaar übernahm 1974, bevor Schmidt-Koenig nach Tübingen ging, seine Taubenschläge am Zoologischen Institut und die dort gehaltenen Tiere (Wiltschко, R. \& Wiltschко, W., mündl. Mitt.).

Doch Kontakte gab es zum Glück für den Botanischen Garten noch auf einer weiteren Ebene. Der langjährige Mitarbeiter des Botanischen Gartens Hans Grasmück, als Gärtnermeister und späterer stellvertretender Technischer Leiter über 40 Jahre tätig gewesen, war wie Schmidt-Koenig Mitglied der Deutschen Dendrologischen Gesellschaft und so kam es auch auf diesem Gebiet zu einer fruchtbaren Zusammenarbeit. Die Gelegenheit, mit einem regelmäßig in Nord-Amerika weilenden Mann Pflanzensamen und Pflanzen zu tauschen, ermöglichte Hans Grasmück zum Wohle des Botanischen Gartens Gehölzraritäten zu erstehen, an die ansonsten kaum heranzukommen gewesen wäre (GrAsmück, mündl. Mitt.). Unter einigem anderen gelangte auf diesem Wege im Jahre 2000 das erste Exemplar von Franklinia alatamaha $\mathrm{zu}$ uns und fand im Revier Nord-Amerika in Frankfurt am Main einen ersten Standort, der nach einigen Jahren allerdings gewechselt wurde, da es sich um einen $\mathrm{zu}$ schattigen Bereich gehandelt hatte. Diese Pflanze war als Absenker zu uns gekom-

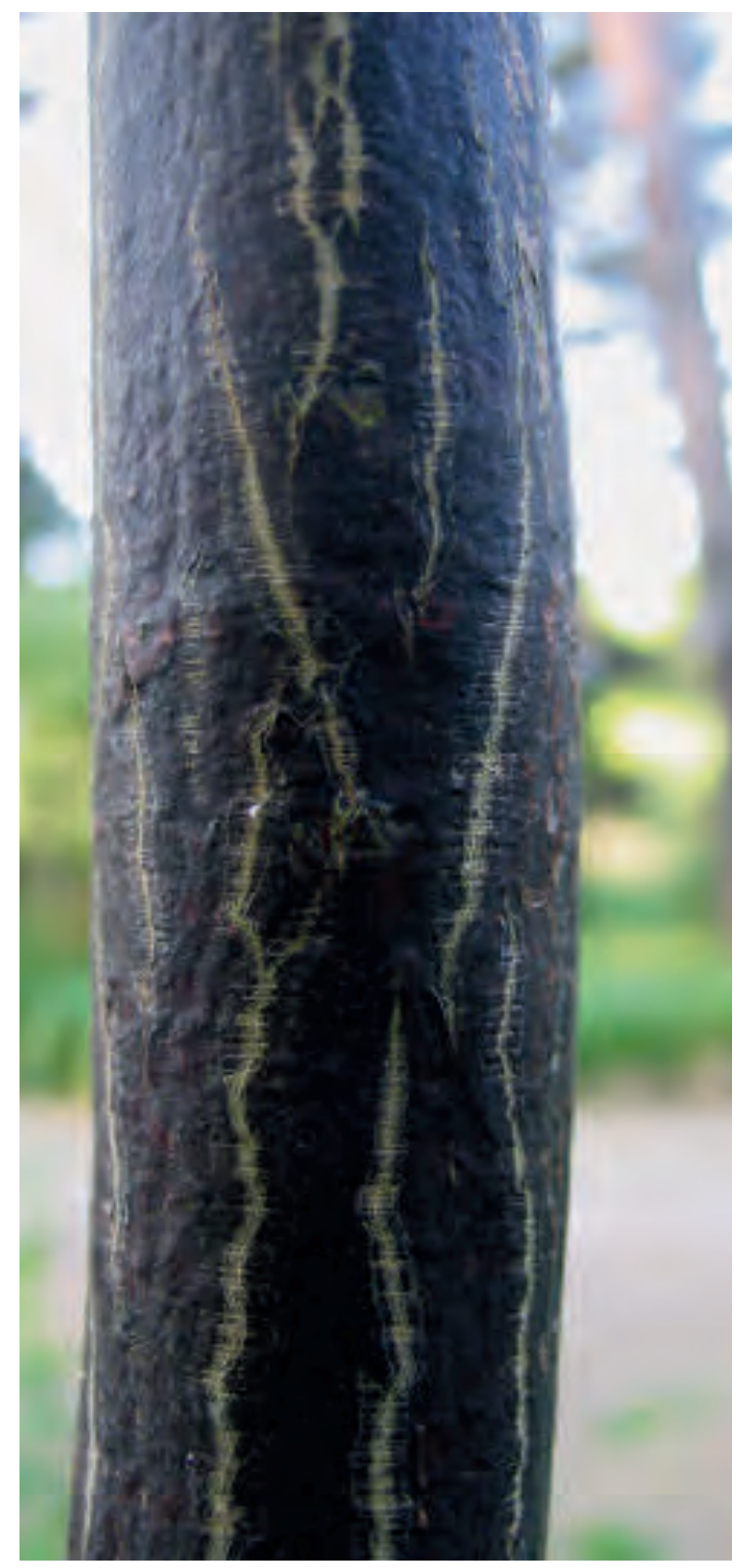

men. Dies bedeutet, dass Schmidt-Koenig seine Pflanze in seinem Arboretum vegetativ vermehrt hatte und einen Zweig zum Bewurzeln gebracht hatte. Ursprünglich stammte die Ausgangspflanze aus Samenvermehrung, der Samen wiederum kam 1975 aus dem SchmidtKoenig wohl bekannten und weltweit berühmten Arnold-Arboretum, dem Botanischen Garten der Harvard-Universität der Stadt Boston im Bundesstaat Massachusetts an der Nordostküste der USA. Es ist davon auszugehen, dass auch die zweite, fast genauso große Pflanze (beide messen ca. $380 \mathrm{~cm}$ und sind 
jeweils 2-stämmig), die wir im Jahr 2012 erhielten, von der Ausgangspflanze von 1975 abstammt. Dieses 2012 vom Botanischen Garten Tübingen bezogene Exemplar gelangte nach dem Tod von Schmidt-Koenig zuerst in die schwäbische Universitätsstadt, da auch dorthin seit langem gute Kontakte seitens der Familie des Verstorbenen bestanden. Man war bestrebt, aufgrund der unsicheren Zukunft des Arboretums in Oberkirch, einige der wertvollsten Gehölze, die noch nicht zu groß waren, an sichere Orte zu bringen, um ihren Fortbestand zu sichern. Nun war aber der Bestand an Franklinien im Botanischen Garten Tübingen schon recht gut, sodass man sich entschloss, die guten Beziehungen der Botanischen Gärten Tübingen und Frankfurt am Main zu nutzen und einen Platz für das wertvolle Gehölz im milden Rhein-Main-Gebiet zu finden (Junginger, B., mündl. Mitt.). Franklinia ist auch im raueren Klima Tübingens absolut winterhart, allerdings gibt es dort im Gegensatz zum Frankfurter Klima regelmäßig im Oktober zur Blütezeit Frühfröste. Das Ergebnis war und ist regelmäßiger Blüh-Ausfall, was in Frankfurt kaum zu befürchten ist. Nun steht auch dieses Tübinger Exemplar bei uns, ganz in der Nähe zur zuerst bezogenen Pflanze und mittlerweile ergänzt durch zwei noch junge Exemplare, die wir im Jahr 2013 käuflich erwarben. Zum Glück für viele sachkundige und

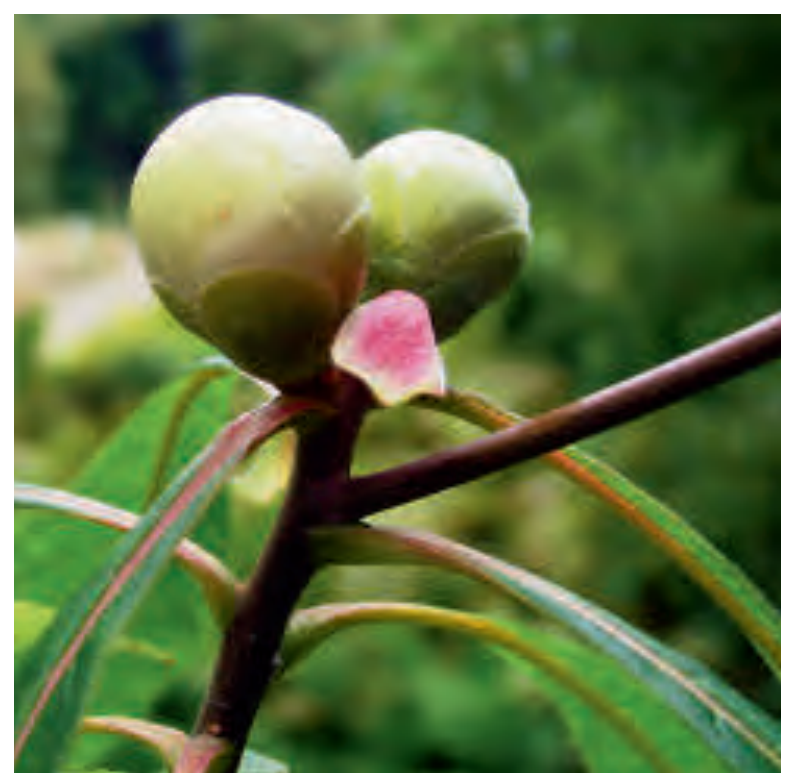

interessierte Gehölz- und Gartenfreunde hat der gärtnerische Fachhandel diese Gehölzart nämlich in die Spezialistensortimente aufgenommen. Damit ist eine weitere Verbreitung gesichert, worüber man nur froh sein kann.

\section{Franklinia alatamaha MARSHALL}

\section{(Theaceae): Zur Geschichte, Botanik} und Ökologie einer fast ausgestorbenen Pflanzenart

Franklinia alatamaha ist eine monotypische Gattung, d.h. die Gattung umfasst nur diese eine einzige Art. Sie ist u.a. nah verwandt mit den Gattungen Camellia, Gordonia ( $G$. alatamaha ist ein Synonym von Franklinia alatamaha) und Stewartia (ERHARdT et al. 2008). Als Mitglied der Teestrauchgewächse wird sie in die Ordnung der Heidekrautartigen (Ericales) gestellt. Grundsätzlich gilt die Unterteilung der Theaceae in Gattungen und Arten als schwierig und wird mitunter noch uneinheitlich gehandhabt, hier werden erst weitere molekulargenetische Untersuchungen weiterhelfen.

Der wissenschaftliche Gattungsname Franklinia wie auch die deutschen Namen Franklinie und FrankLIN-Baum beziehen sich auf den amerikanischen Politiker, Schriftsteller und Naturforscher Benjamin Franklin (1706-1790). Man schrieb das Jahr 1765, als Vater John Bartram (1699-1777) und sein Sohn William (1739-1823), beide Botaniker und Naturforscher, an der Mündung des Altamaha River in Georgia (USA) einen ihnen unbekannten Baum entdeckten, der als botanische Neuentdeckung galt. William Bartram nahm auf einer späteren Reise zu diesem Standort Samen des Baumes mit und erzielte so einige Nachkommen, die er zu Ehren seines Freundes Benjamin Franklin auf den uns heute geläufigen Namen Franklinia taufte. Der Fluss Altamaha hieß bei den dort ansässigen Indianern des Volkes der Cherokee „alatamaha“, dies erwählte William Bartram als Art-Epitheton. So entstand die heute anerkannte wissenschaftliche Bezeichnung, die seit 1785 Gültigkeit besitzt. Franklinia alatamaha steht auf der Liste der IUCN (Red List of Threatened Species; International Union for Conversation of 
Nature and Natural Resources; Internationale Rote Liste der gefährdeten oder ausgestorbenen Pflanzenarten) und gilt seit 1790 bzw. 1803 als ausgestorben. Als vermutliche Ursache des Verschwindens der Art wird am häufigsten ein durch Baumwolle eingeschleppter Pilz genannt, aber wissenschaftlich gesichert ist dies nicht. Den Bartrams ist es auf jeden Fall zu verdanken, dass diese Gehölzart heute wieder mehr und mehr die Gärten und Parks verschönert. Sie pflanzten die ersten Sämlinge in ihren Garten in Pennsylvania ca. 1770 und gaben bereitwillig deren Nachkommen weiter. Bei einer 1998 weltweit durchgeführten Zählung („Franklinia Census“) wurden ca. 2000 Exemplare in Privatgärten, Parks und Botanischen Gärten ermittelt, die allesamt Nachkommen von den Bäumen sind, die die Bartrams einst pflanzten. Heute dürfte diese Zahl deutlich höher liegen. Und um noch ein weiteres Mal auf Schmidt-Koenig zurückzukommen: Er hatte mehrere Anträge auf Auswilderung der Art in den ehemaligen Heimatgebieten in Georgia gestellt, die aber alle von den zuständigen US-Behörden abgelehnt wurden (Wiltschкo, R. \& Wiltschкo, W., mündl. Mitt.).

Franklinia ist ein aufrechter, sommergrüner Strauch oder kleiner Baum bis 10 m Höhe. Ein Rückschnitt in der Gartenkultur, z.B. um einen mehr verzweigten Habitus zu erzielen, verbietet sich. Das Gehölz verfügt über keine schlafenden Augen, aus denen heraus neue Triebe entstehen könnten. Die wechselständig angeordneten Blätter sind einfach, länglich oval bis lanzettlich und $12-15 \mathrm{~cm}$ lang, schwach gesägt, zugespitzt, mit schmaler keilförmiger Basis, entfernt gesägt, oberseits glänzend grün und unterseits behaart. Im Herbst schmückt

Abb. 5 (Seite 66): Blütenknospe.

Abb. 6 (oben): Geöffnete Blüte einer Franklinie.

Abb. 7 (Mitte): Blick in die Blüte mit ihren zahlreichen Staubblättern.

Abb. 8 (unten): Staubblätter im Detail.
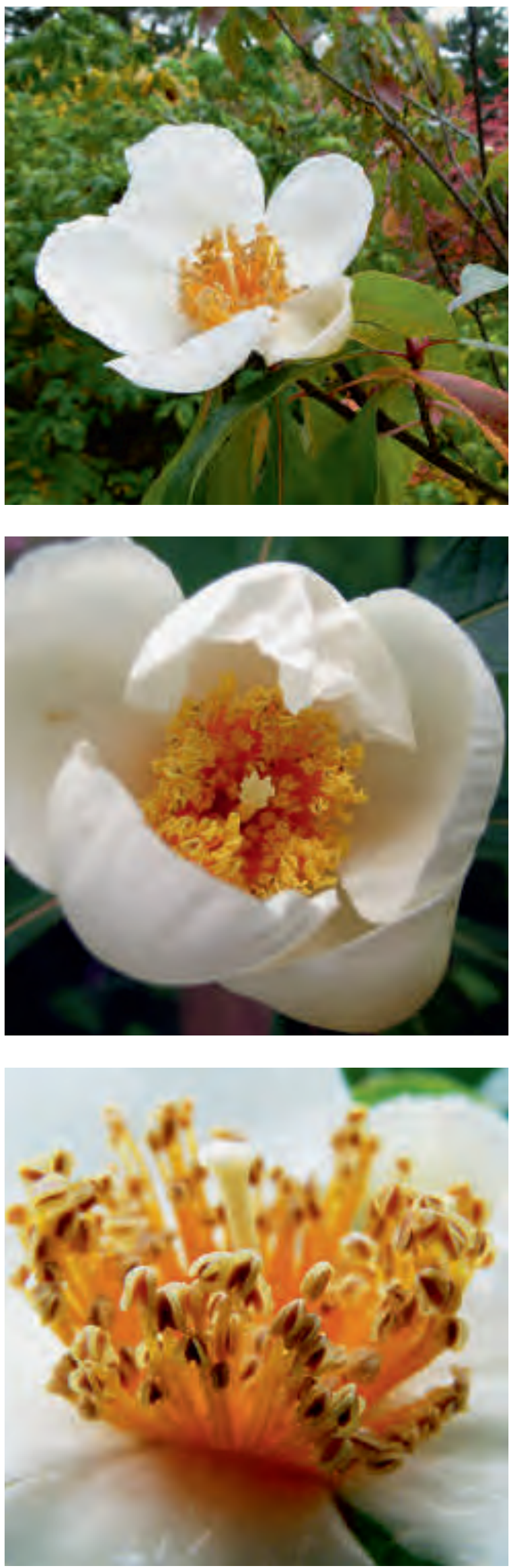
sich die Franklinie mit oft leuchtend roten Blättern. Die Rinde des Gehölzes ist dünn und glatt, mit länglichen hellen Streifen gezeichnet, die Zweige sind olivgrün gefärbt. Die weißen, den Scheinkamelien ähnelnden, leicht orangenartig duftenden Blüten sind $6-8 \mathrm{~cm}$ breit, becherförmig, achselständig und haben eine 5-zählige Blumenkrone. Die einzelnen Kronblätter sind rundlich-eiförmig und umschließen die zahlreichen goldgelben Staubblätter und den einen oberständigen 5-fächrigen Fruchtknoten. Aus diesem entwickeln sich kugelrunde, bis $2 \mathrm{~cm}$ breite holzige Kapseln, die bei Samenreife unten und oben aufspringen. Jedes der 5 Fächer enthält bis zu 8 kantige, bis $1 \mathrm{~cm}$ große Samen (KrüsSMann 1974, Rohloff \& Bärtels 2006). Die Blütezeit liegt zwischen September und Oktober, was einerseits wunderbar ist, denn zu dieser späten Jahreszeit blühen nicht mehr viele Gehölze, und diese wunderschöne und sehr auffällige Blüte erfreut jedes Herz, das ihrer ansichtig wird. Andererseits führt die späte Blütezeit, auch wenn das Gehölz an sich vollkommen winterhart ist, in etwas raueren Lagen zum garantierten Absterben der Blüten, wenn es zu Frühfrösten kommt. Dies ist z. B. bei unseren Kollegen in Tübingen recht regelmäßig der Fall und schmälert den Genuss an der Pflanze erheblich. Hier bestehen Parallelen zu den Magnolien, die ebenfalls, allerdings durch späte Fröste im Frühjahr, immer mal wieder in der Blütezeit geschädigt werden. In Frankfurt am Main ist die Gefahr durch Frühfröste recht gering, sodass wir hier einen klimatischen Vorteil haben. Zur Samenbildung sind die Früchte im Botanischen Garten Frankfurt am Main aber auch noch nicht gelangt, soweit die Erinnerung der Gärtner und Gärtnerinnen zutreffen.

Der ehemals heimische Standort der Franklinie in den Küstenebenen des südöstlichen Georgia in McIntosh County waren die Ufer des Alatahama River auf sauren humosen Sanddünen, die als nährstoffreich und gut versorgt gelten können. Naturgemäß waren diese Standorte frisch bis feucht und eher sonnig bis lichtschattig als halbschattig, was sich mit unseren Erfahrungen im Botanischen Garten deckt. Ganz sicher handelt es sich um eine Art, die eher als anspruchsvoll und mit enger Standortsamplitude ausgestattet zu gelten hat (Kiermeier 1995).

Trockenheit wird schlecht vertragen, die Hitzeverträglichkeit bei ausreichender Bodenfeuchte ist aber sehr gut und befördert in heißen Sommern das Ausreifen des Holzes und damit die Erhöhung der Verträglichkeit für tiefe winterliche Temperaturen. Dies alles lässt sich für den Gehölzfreund in Mitteleuropa auf den Gartenstandort bezogen so zusammenfassen: ein guter, humoser, leicht saurer Boden in wenigstens lichtschattiger, gern windgeschützter und warmer Lage mit ausreichend guter Wasserversorgung, aber bitte ohne stauende Nässe und Kalkgestein im Untergrund - dann sollte ein Erfolg bei der Gartenkultur gelingen und zu dieser kann angesichts der Seltenheit, Schönheit und Besonderheit dieser Pflanzenart nur zugeraten werden.

\section{Literatur}

Erhardt, W., Götz, E., Bödeker, N. \& Seybold, S. 2008: Der große Zander, Enzyklopädie der Pflanzennamen, Bd. 1: Familien und Gattungen. - Stuttgart. Kiermeier, P. 1995: Die Lebensbereiche der Gehölze eingeteilt nach dem Kennziffernsystem. - Pinneberg. Krüssmann, G. 1977: Handbuch der Laubgehölze, Bd. II. - Berlin, Hamburg.

Roloff, A. \& Bärtels, A. 2006: Flora der Gehölze. Stuttgart.

\section{Internetseiten}

Ganzhorn, J. \& Hoffmann, R. 2010: Nachruf auf Klaus Schmidt-Koenig von seinen Schülern aus dem Kupferhammer.

http://www.dzg-ev.de/de/publikationen/mitteilungen_ zoologie/2010/2010_75_nachruf_schmidt_koenig.pdf (abgerufen am 25. April 2014).

http://de.wikipedia.org/wiki/Brieftaube

(abgerufen am 25. April 2014).

http://de.wikipedia.org/wiki/Franklinia

(abgerufen am 25. April 2014).

Stevens, P. F. (2001 onwards). Angiosperm phylogeny website. http://www.mobot.org.MOBOT/research/APweb/ (abgerufen am 25. April 2014).

Tropicos.org. Missouri Botanical Garden.

http://www.tropicos.org (abgerufen am 25 April 2014.

World Conservation Monitoring Centre 1998.

Franklinia alatamaha. In: IUCN 2013. IUCN Red List

of Threatened Species. Version 2013.2. <www.iucnredlist. org>. http://www.iucnredlist.org/details/30408/0

(abgerufen am 25 April 2014) 